\title{
Erratum: Magnetic susceptibility in three-dimensional nodal semimetals [Phys. Rev. B 93, 045201 (2016)]
}

\author{
Mikito Koshino and Intan Fatimah Hizbullah \\ (Received 10 May 2019; published 20 May 2019)
}

DOI: 10.1103/PhysRevB.99.209903

The authors regret that they inadvertently omitted the citations of papers which studied the magnetic susceptibility of the Dirac electrons before this article. The orbital magnetic susceptibility of the Dirac Hamiltonian was theoretically investigated for bismuth [1] where the logarithmic behavior was found. Equation (43) obtained in the original paper is essentially equivalent to that in Ref. [1]. The singular magnetic susceptibility of the two-dimensional Dirac electrons mentioned in the Introduction was studied in Refs. [2,3]. The diamagnetism of Dirac electrons was also extensively argued in more recent works $[4,5]$.

[1] H. Fukuyama and R. Kubo, J. Phys. Soc. Jpn. 28, 570 (1970).

[2] H. Fukuyama, J. Phys. Soc. Jpn. 76, 043711 (2007).

[3] A. Kobayashi, Y. Suzumura, and H. Fukuyama, J. Phys. Soc. Jpn. 77, 064718 (2008).
[4] Y. Fuseya, M. Ogata, and H. Fukuyama, J. Phys. Soc. Jpn. 81, 093704 (2012).

[5] Y. Fuseya, M. Ogata, and H. Fukuyama, J. Phys. Soc. Jpn. 84, 012001 (2015). 